\title{
Keyword Index Volume 25 (2012)
}

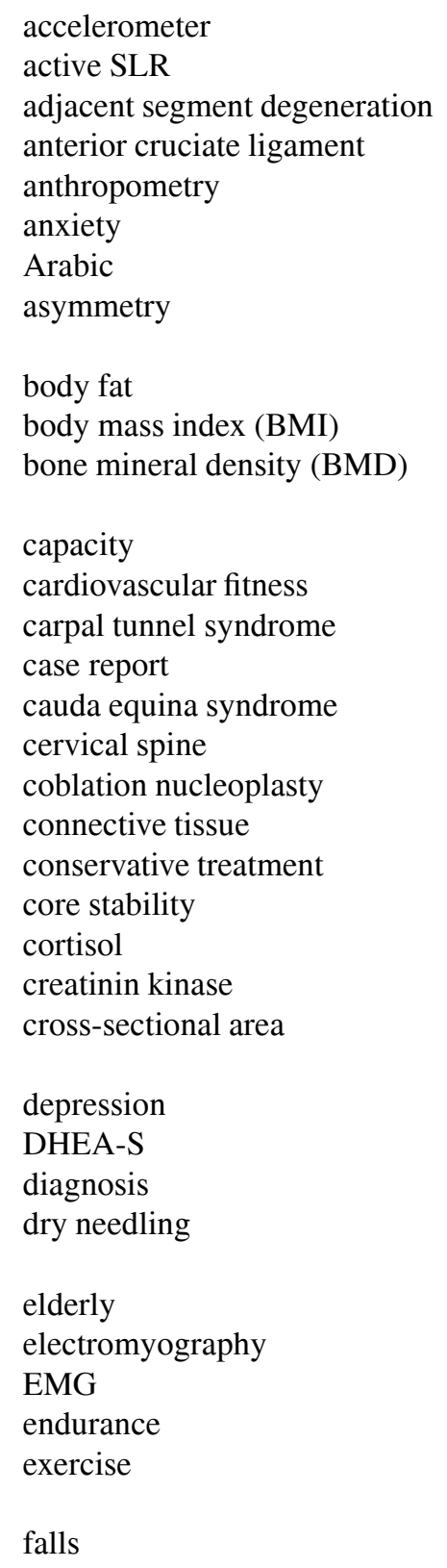

FFT

fibromyalgia 245

flat feet $\quad 21$

functional disability 149

gait symmetry 269

glucosamine sulfate 275

ground reaction force $\quad 21$

handrails $\quad 33$

heights 33

hydrotherapy 225

idiopathic inflammatory myopathy 231

instability 123

internal oblique 239

isokinetic exercise $\quad 81$

isometric contraction $\quad 95$

kinesio tape $\quad 187$

knee cartilage $\quad 275$

knee joint 117

knee osteoarthritis 193

landing $\quad 21$

lateral epicondylitis (tennis elbow) 131

likelihood ratios 215

LLLT 13

low back pain $\quad 73,123,201$

lumbar disc herniation 1

lumbar multifidus muscle $\quad 171$

lumbar segmental instability $\quad 149$

magnetic resonance imaging 275

manual therapy 225

mechanical neck disorder $\quad 47$

Mini-Osteoporosis Quality of Life Questionnaire 89 motion analysis $\quad 21$

mud pack therapy 193

multiplied linear dimension $\quad 171$

$\begin{array}{ll}\text { muscle } & 67\end{array}$ 
muscle strength

muscle thickness

muscle timing

musculoskeletal physiotherapy

myofascial pain

myofascial pain syndrome

neck movement

neurobrucellosis

neuroplasticity

obesity

osteitis pubis

osteoarthritis

outcomes

pain

pain pathology

pain terminology

pelvic

percentage of change in

performance

physical activity

physical fitness

physiotherapy

polymyositis

postmenopausal osteoporosis

postural stability

posture

pressure algometry

pressure pain threshold

progressive resistive exercise

quadriceps

quality of life

rehabilitation

relative contribution ratio

reliability

risk factors

sacroiliac pain

scapular depression syndrome
Keyword Index Volume 25 (2012)

123 scapular elevation taping $\quad 187$

239 semispinalis capitis 67

27 sensitivity 215

103 sensorineural hearing loss 157

285 shoulder 13

261 single-strap bag 269

sit-to-stand movement 33

47 size 67

157 sound 117

$291 \quad$ spastic paraparesis $\quad 157$

specificity 215

143, 253 sphincter dysfunction 1

225 spine 109, 161, 177

$89,117,143,275 \quad$ stabilization $\quad 73$

177 stabilization exercise 109

stabilizing exercise 149

$13,55,149,245,261 \quad$ straight leg raise test 215

103 straight leg raising test 215

103 strap-length 269

161 stretching program $\quad 161$

239 systematic review 215

177

177 teachers 5

253 temporomandibular joint 285

13,73 tendonitis 13

231 three-dimensional electromagnetic 47

55 torque 95

109 transversus abdominis 239

55 triceps surae 95

285 trunk motion 269

187 the 171

81 ultrasonography 171

$\begin{array}{ll}\text { ultrasound } & 13 \\ \text { ultrasound imaging } & 67\end{array}$

81 ultrasound imaging 67

5, 193 upper limb tension test 209

$73,177,231$

239 validation 89

201,209 validity 201

5 vertebral fracture $\quad 55$

27 work 161

187 work-related pain 5 